\title{
A New Method for Knot Placement of B-Spline Curve Approximation and Interpolation
}

\author{
Wei Wang ${ }^{1, a}$, Fazhong Shi ${ }^{1, b}$, Gang Zhao ${ }^{1, \mathrm{c}}$ and Xiaoxiao $\mathrm{Du}^{1, \mathrm{~d}}$ \\ ${ }^{1}$ School of Mechanical Engineering \& Automation, Beihang University, Beijing 100191, PR China \\ ajrrt@buaa.edu.cn, bshifzh@buaa.edu.cn, 'zhaog@buaa.edu.cn, dduxxhf@163.com
}

Keywords: B-spline curve approximation, knot placement, curve fitting

\begin{abstract}
A new knot placement method for B-Spline curve fitting to a set of ordered points is presented in this paper. It is a unified explicit formula that applies to both approximation and interpolation. The experiments designed by authors show that this method is effective and it has the advantage of keeping bilateral symmetry features inherently in the data points unchanged in the resulting approximation cure, which means it can reflect more essential shape features of original data than the existing ones, and has the potential of being widely used in practices. This knot placement method is simple, convenient and pervasive, while the curves obtained by it can provide more flexible options for the shape design work.
\end{abstract}

\section{Introduction}

B-Spline curve approximation is one of the classic problems that have been established in the field of Computer Aided Geometric Design (CAGD) [1, 2]. Nonetheless B-Spline curve approximation is still an essential operation in many applications. For example, large amounts of data created in reverse engineering and various processes of engineering design must be approximated by smooth B-Spline curves. With the increasing of the computer speed and the development of reverse engineering, from the mid-1990s, B-Spline curve approximation has become a research hotspot in CAGD field, quite a lot of research literatures emerged in the publications [3,4,5] and research progress has been continuously made. Piegl and Tiller [2] pointed out that approximation is more difficult than interpolation. In interpolation, the number of control points is automatically determined by the chosen degree and the number of data points, so the knot placement is straightforward and there is no curve or surface error to be checked. In approximation, a curve/surface error bound $E$ is input along with the data points to be fitted. It is usually unknown in advance how many control points are required to obtain the desired accuracy $E$, hence approximation methods are generally iterative for why it is more time-consuming than interpolation. The important issue in B-Spline curve approximation is to reduce the number $n+1$ of control points while keeping the desired accuracy, generally requiring an iterative process that adjusts the number $n+1$ to maintain an error bound. Least-squares curve fitting is often used as one step in this iterative process and equations need to be solved in this step.

Giving the appropriate knot placement is essential for the success of the B-Spline curve approximation. Since the 1990s, many scholars have explored this issue unremittingly. Despite some progress, no important breakthrough being made, and the algorithms has been becoming more and more complicated. How to place knot(s) is still without perfect solution [6].

In this paper, based on Piegl and Tiller's work [2, 3, 4], authors propose a unified averaging technique for both approximation and interpolation. It is demonstrated by experimental results that this technique is simple, effective and fast.

\section{The Previous Knot Placement Techniques and Algorithms}

So far, there is a variety of knot placement methods:

(1)The average technique (AVG) + the knot placement technique (KTP) 
If the interpolations of data points $\boldsymbol{q}_{i}$ in interpolating spline curve occur at the knots, a singular system of equations may be encountered in the calculation. Thus, Piegl and Tiller recommended the following method [2]:

They pointed out that the placement of knots should reflect the distribution of the parameter values $\tilde{u}_{i}$ of the data points, the internal knots in definition domain can be decided as follows:

$$
u_{k+j}=\left\{\begin{array}{lr}
\frac{1}{k} \sum_{i=j}^{j+k-1} \tilde{u}_{i}, & m=n \\
(1-\alpha) \tilde{u}_{l-1}+\alpha \tilde{u}_{l}, & m>n \quad(j=1,2, \cdots, n-k) \\
\text { here } \ell=\operatorname{int}(j c), c=\frac{m+1}{n-k+1}, & \alpha=j c-\ell
\end{array}\right.
$$

This is called the averaging technique (AVG) for $m=n$, and the knot placement technique (KTP) for $m>n$, wherein $k$ is the degree of the fitting curve, $n+1$ is the number of control points and $m$ that of the data points.

(2) The new knot placement technique (NKTP)

Piegl and Tiller noted that in AVG+KTP method the treatment for approximation is different from that of interpolation, i.e. the two treatments are not unified. And they also noticed that a stable system of equations cannot always bring acceptable approximating curve solution. For example when $m>n$ and $n$ is nearly equal to $m$, the distribution of data points and parameter values are sensitive, it is possible to generate an unwanted result, particularly in the vicinities of the two end points. In order to avoid the existing stability problem they suggested another technique that is called new knot placement technique (NKTP) in [3].

(3)The knot removal technique

Piegl and Tiller [2] mentioned another approximation scheme: (a) start with the maximum or "many" control points, enough for the first approximation to satisfy the given approximation error bound E; (b) fit a curve to the data points, using a global fit method; (c) check if the deviation is satisfied everywhere; (d) if $E$ is no longer satisfied return the previous curve, else reduce the number of control points and go back to (b). In order to reduce the number of control points, the knot removal technique [7, 8] is used. The curve after knot removal differs generally from the original curve, with both control points and knots changed but the impact of removing a knot is local. However, the scheme is much more expensive than those that are previously mentioned, and undesirable results may be generated for a large number of dense data points especially when with noise.

There are also other methods like the method of dominant points and feature points [6, 10], genetic algorithm $[10,11]$ and so on. These methods are usually effective for some occasions but as a general approach, they appear complex and consume too much computation resources. In the design community some methods with direct geometric meaning and simplicity are more preferred.

\section{The Proposed Knot placement Techniques}

The Modified Knot Placement Technique (MKPT). Of all the above algorithms, the AVG for interpolation knot placement is determined by using a very simple arithmetic average of consecutive $k$ ( $k$ is the degree of approximation curve) parameter values, which is intuitive and easy for implementation. The advantage of AVG is unanimously recognized in CAGD academia and industrial applications. So we associate it with the knot placement for approximation to explore whether or not approximation problem can find a similarly simple formula.

At first there is the need to take into consideration the symmetry problem. Here when we talk about the symmetry we only mean the bilateral symmetry which widely exists in both human culture and natural phenomena. In human history, the symmetry ornaments seem to represent perfect order, beauty, and divine harmony, while in the natural world higher animals seem to use bilateral symmetry to solve the problem of optimal mobility with simultaneous balance of forces [12]. The symmetric form is utilized in the design of many industrial products for both aesthetic and 
functional purposes, including spacecrafts, airplanes, high-speed trains, cars, almost all vessels and warships except aircraft carrier. Thus we think the curve approximation method is more complete if with the ability of keeping the symmetry feature originally in the given data points, by which the potential function of the approximated subject can be fully exploited.

The question now is how to ensure that resulting B-Spline approximation curve has the same symmetry if the given data points are symmetric. As the parameterization of symmetric data points i.e. the resulting parameter value is symmetric, to guarantee the approximation curve has symmetry, the knot values in knot vector must also be symmetric. For this reason, we suggest to modify (1) as follows:

$$
u_{k+j}=\left\{\begin{array}{lr}
\frac{1}{k} \sum_{i=j}^{j+k-1} \tilde{u}_{i}, & m=n \\
(1-\alpha) \tilde{u}_{l-1}+\alpha \tilde{u}_{l}, & m>n \quad(j=1,2, \cdots, n-k) \\
\text { here }: l=\operatorname{int}(j c), c=\frac{m+2}{n-k+1}, & \alpha=j c-l
\end{array}\right.
$$

Only the numerator $m+1$ in Eq. 1 is changed into $m+2$ in Eq. 2 results in that the approximation curves inherit the symmetric property of data points. And the property is independent of the parity of control points, as shown in Fig. 1. However when $n$ is nearly equal to $m$, the problem of generating unacceptable curve still exists, as shown in Fig. 2.

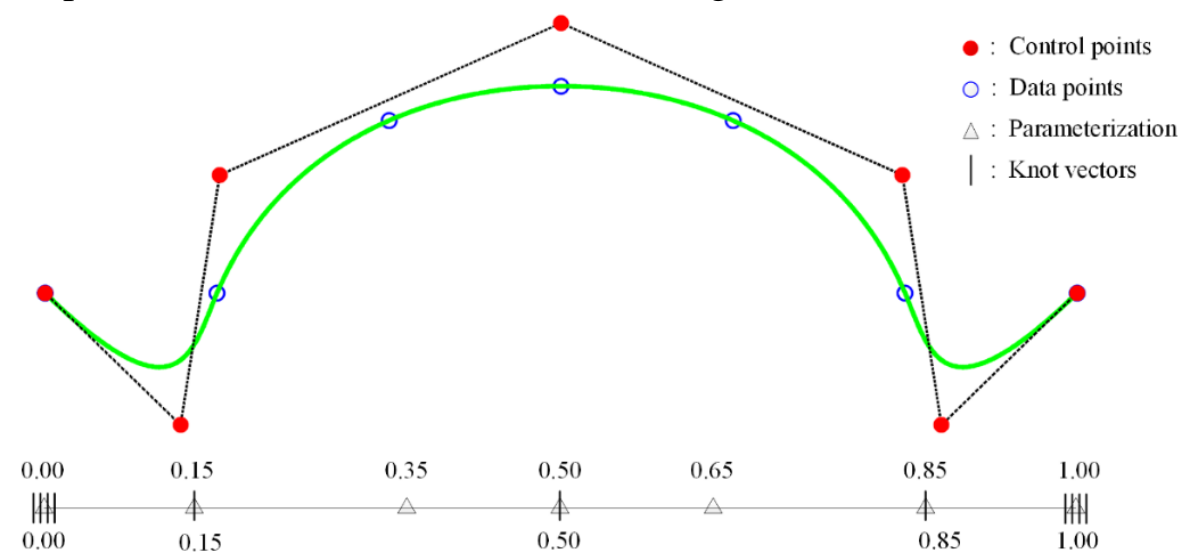

(a) Interpolation curve $k=3, m=6, n=6$

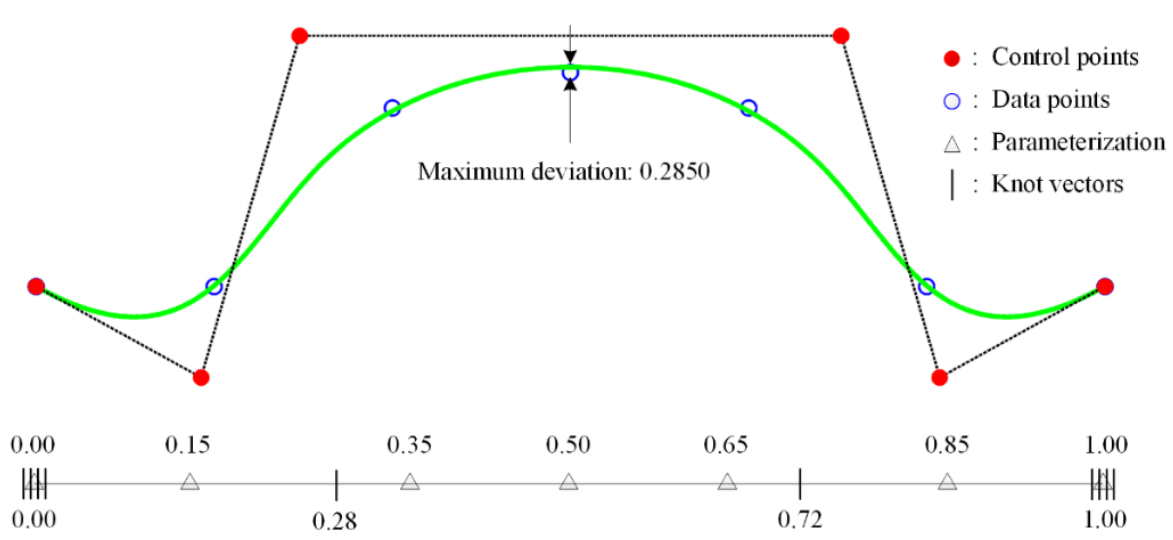

(b) Approximation curve $k=3, m=6, n=5$

Fig. 1 The approximation curve generated by MKTP inherits the symmetry of the data points 


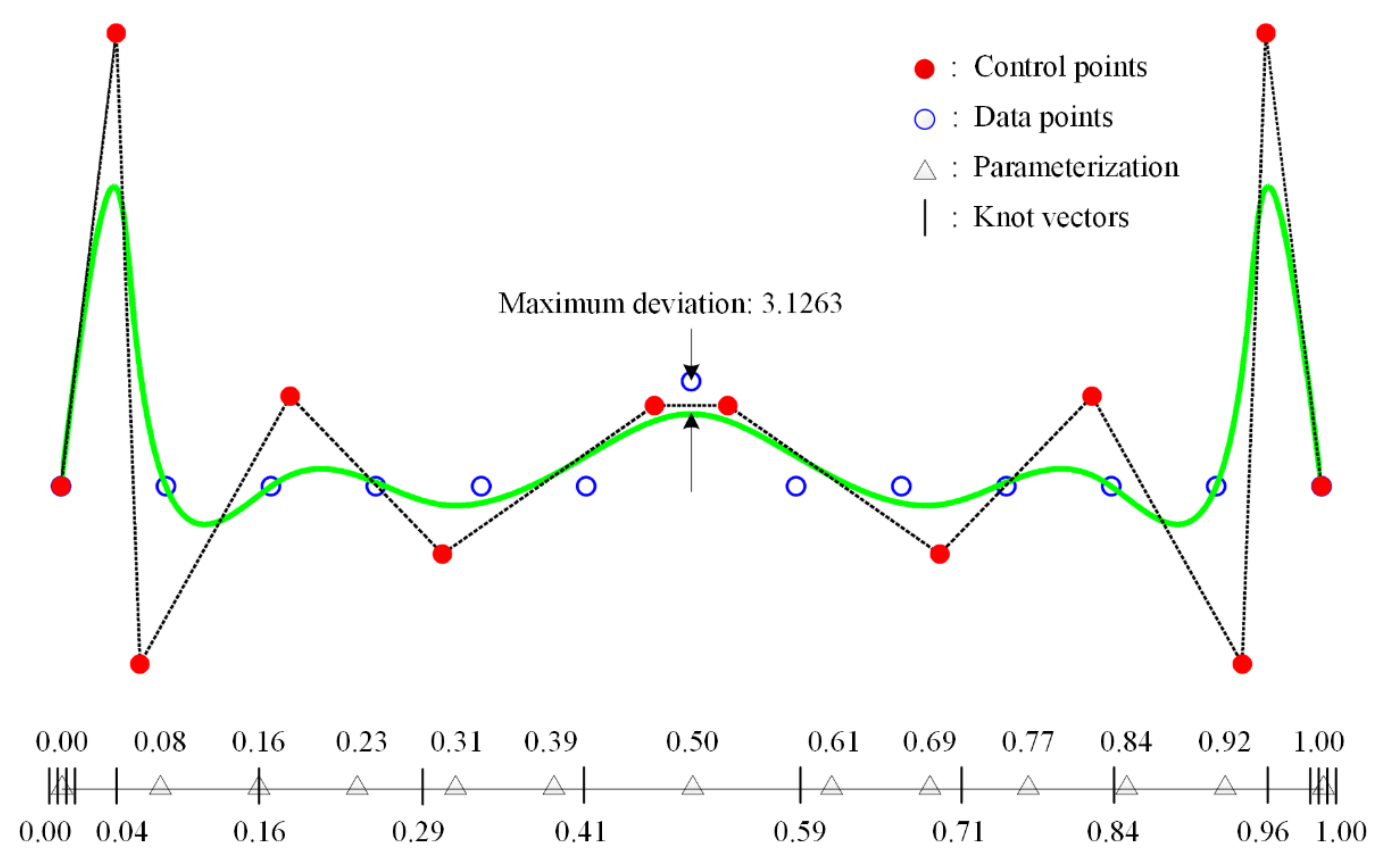

Fig. 2 MKPT solves symmetry issue, but when $n$ is nearly equal to $m$, generating unacceptable curve problem still exists $(k=3, m=12, n=11)$

The Proposed Unified Average Technique (UAVG). The MKTP method is successful in solving symmetry problem, but there is still non-uniform problem, i.e. the formula for approximation is different from that for interpolation. Both NKTP and KTP have symmetry problem: the interpolation curves generated by symmetric data points maintain symmetry, but the approximation curves lose symmetry. And NKTP does not give explicit expression. Therefore, authors suggest the following unified averaging technique (UAVG):

$$
\begin{cases}u_{j}=0, & j=0,1, \cdots, k \\ u_{k+j}=\frac{1}{m-n+k} \sum_{i=j}^{m-n+k-1+j} \tilde{u}_{i}, & j=1,2, \cdots, n-k \\ u_{n+j}=1, & j=1,2, \cdots, k+1\end{cases}
$$

Hence the internal knot formulas can be expressed as: the parameter value of the $j$ th internal knot is equal to the arithmetic average of parameter values of the $m-n+k$ data points from the $j$ th. When $m=n$, we obtain the formula of the internal knots for interpolation given by AVG, i.e. this method contains the internal knot calculation formula for interpolation in Eq. 1. Thus the Eq. 3 and the internal knot calculation for interpolation in Eq. 1 are unified. The practices show that this simple and quick method not only achieves unified treatment for both approximation and interpolation, but also solves the symmetry problem of KTP and NKTP.

Examples of Knot Placement Using UAVG. In this part we give experiments to test the UAVG and discuss its properties. They are shown in Fig. 3-6. From all the experiments, it shows that knot placement using Eq. 3 results in symmetry keeping approximation curve and can be used for various occasions that often encountered in practices, such as collinear data and when the number of control points is nearly or exactly equal to that of data points. These properties can guarantee the method proposed have pervasive application opportunities in real CAD work. Here it's no necessary to list more experiment results one by one. 


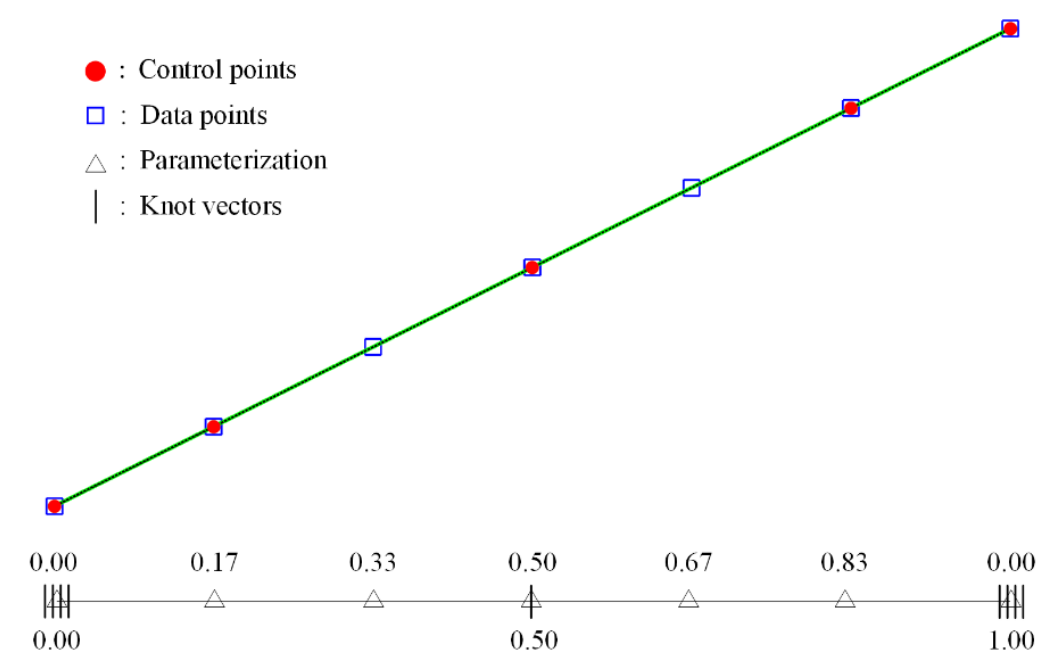

Fig.3 Collinear data points generate straight line $(k=3, m=6, n=4)$

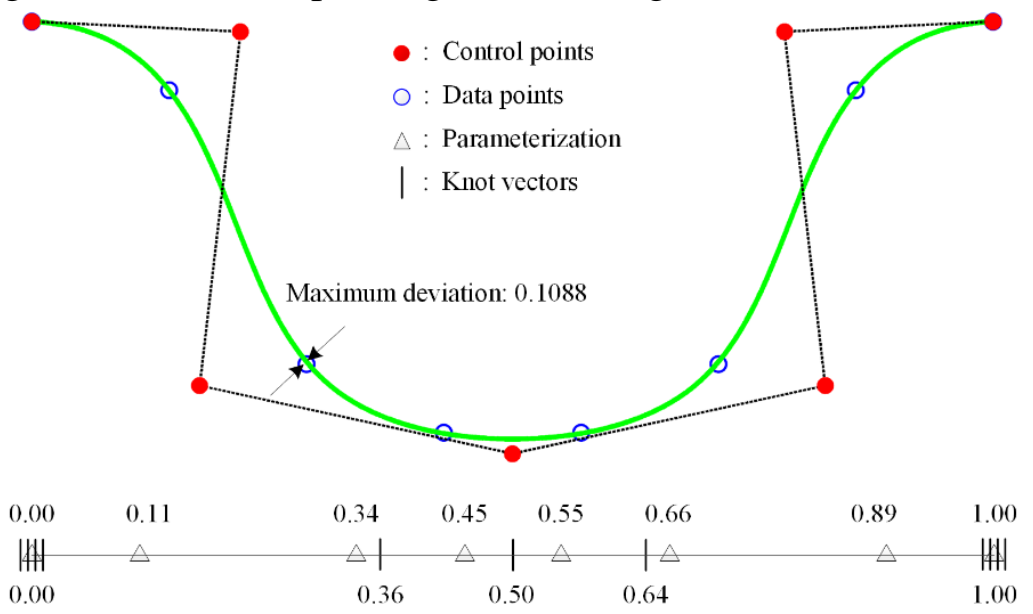

Fig. 4 Symmetric data points generate symmetric curve $(k=3, m=7, n=6)$

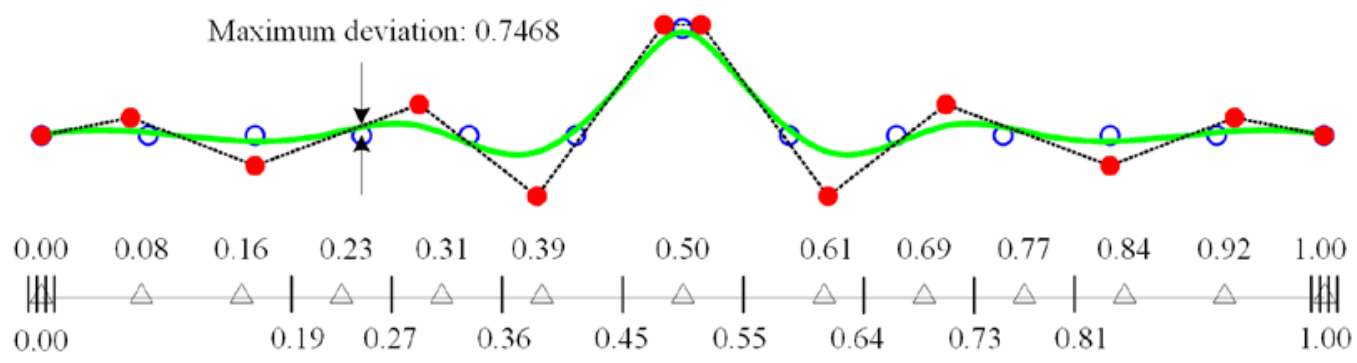

Fig. 5 When $n$ is nearly equal to $m$, it still yields curve of good quality ( $k=3, m=12, n=11$ )

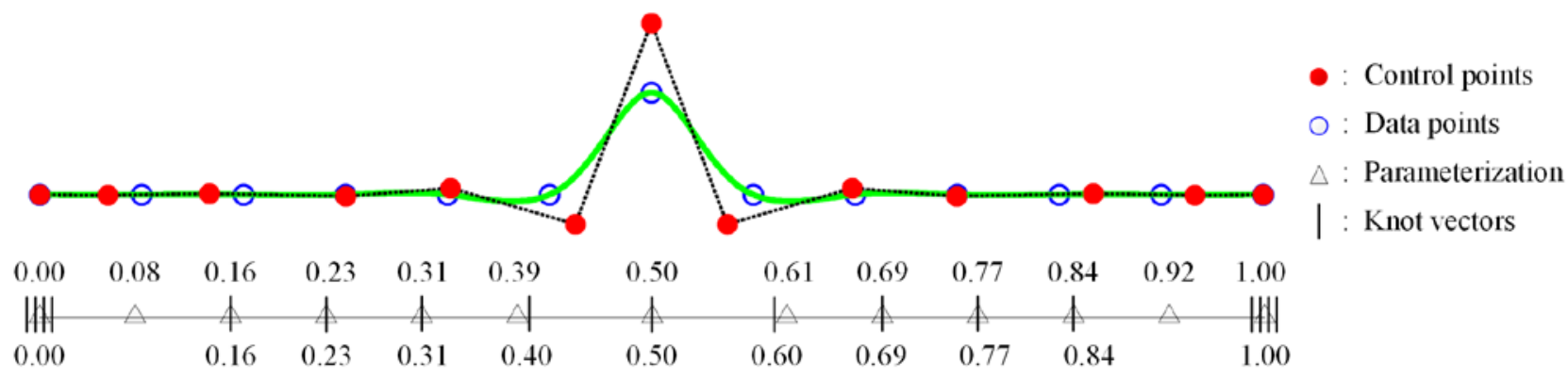

Fig. 6 When $m=n$, B-Spline curve approximation becomes B-Spline curve interpolation $(k=3, m=12$, $n=12)$ 


\section{Conclusion}

In the case that strict interpolation is not required, to approximate ordered data points by using B-Spline curve in least squares sense not only reduces the definition data, but also reaches the effect that approximation curve is smoother than interpolation curve. A difficult problem in B-Spline curve approximation is the knot placement. Based on AVG and KTP, authors propose a unified approach UKTP to determine the knot vector for interpolation and approximation, and give a unified explicit formula for knot placement, by which the symmetry property in original data points can be kept in the final approximation curve. Because of UAVG's simplicity and more shape keeping characteristic, it's a promising algorithm and worthy of being popularized in curve \& surface approximation for design work in industrial practices.

\section{Acknowledgement}

The authors would like to acknowledge the support by the Natural Science Foundation of China (Project NO. 51305016).

\section{References}

[1] Hoschek J, Lasser D. Fundamentals of computer aided geometric design. Wellesley: A K Peters; 1993.

[2] Piegl L,Tiller W. The NURBS book. New York: Springer; 1997.

[3] Piegl L, Tiller W. Least-Squares B-Spline Curve Approximation with Arbitrary End Derivatives. Engineering with Computers, 16 (2000); pp109-116

[4] Piegl L, Tiller W. Surface approximation to scanned data. The Visual Computer, 167 (2000); pp386-395.

[5] Razdan A. Knot placement for B-Spline curve approximation. Report, 1999. Arizona State University. http://citeseer.ist.psu.edu/398077.html.

[6] Xu Jin, Ke Yinglin, Qu Weiwei. B-Spline curve approximation on feature points automatic recognition Journal of mechanical engineering, Chinese, 4511 (2009); pp212-217.

[7] Lyche T, Morken K. Knot removal for parametric B-Spline curves and surface. Computer Aided Geometric Design, 4 3(1987); pp217-230.

[8] Tiller W. Knot removal algorithms for NURBS curves and surfaces. Computer Aided Design, 24 8(1992); pp 445-453.

[9] Park H. B-Spline surface fitting based on adaptive knot placement using dominant columns. Computer Aided Geometric Design, 433 (2011); pp258-264.

[10] Holland, J. H. Adaptation in Natural and Artificial Systems. University of Michigan Press, Ann Arbor, MI. 1975.

[11] Holland J H. Adaptation in Natural and Artificial Systems. 2nd edn. MIT Press, Cambridge, 1992.

[12] Klaus Mainzer. Symmetry and Complexity: The Spirit and Beauty of Nonlinear Science. World Scientific Publishing Company, Singapore, 2005. 